\title{
Some Properties of Relative Regularity and Compactness
}

\author{
Genglei Li \\ College of Science, Tianjin Polytechnic University \\ Tianjin 300160, China \\ E-mail: lglxt@126.com \\ Yunxia Zhang \\ College of E \& A, Hebei Normal University of Science and Technology \\ Qinhuangdao 066004,China \\ E-mail: xyz4418@sina.com \\ Huidong $\mathrm{Wu}$ \\ College of Business, Hebei Normal University \\ Shijiazhuang 050000, China \\ E-mail: xtlqjz@sohu.com
}

\begin{abstract}
In this paper, some relative topological properties were studied, especially including relative regular and relative countable1-paracompact and the property of countable1-paracompact under the perfect mapping was also discussed.
\end{abstract}

Keywords: $\mathrm{X}$ is regular, $\mathrm{X}$ is lindeloff, $\mathrm{Y}$ is regular in $\mathrm{X}, \mathrm{Y}$ is countable1-paracompact in $\mathrm{X}$

\section{Introduction}

Relative topological properties are extension of classic topological invariants.In 1989, the relative topological properties were discussed by A.V.Archangel'skii and H.M.M.Genecli in (A.V.Arhangel'skii. 1996), and A.V.Arhangel'skii gave the first systematic text on relative topological properties in 1996. In recent years, some further new results of the relative topology were obtained respective by A.V.Arhangel'skii, J.Tartir and W.Just, O.Pavlov and M.Matveer, I.Yaschenko, V.V.Tkachuk, M.G.Tkachenko and R.G.Wilson, etc.

In my paper, some relative topological properties were studied and some results were given.

\section{The properties of relative topology}

$\mathrm{X}$ is a space, $Y \subset X$, the concept of $\mathrm{X}$ is regular, lindeloff were introduced in (R.Engelking, 1997) and the concept of $\mathrm{Y}$ is regular in $\mathrm{X}$ and the definition of countable1-paracompact was respectively introduced in (A.V.Arhangel'skii. 1996) and (Gartside P and Aneirin G, 2000) . In this part, some properties of them were discussed, and I gave two results.

Definition $2.1 \mathrm{X}$ is regular: If for each $\mathrm{y}$ of $\mathrm{X}$ and each closed subset $\mathrm{p}$ of $\mathrm{X}$, such that $y \notin p$, there are disjoint open subsets $\mathrm{u}$ and $\mathrm{v}$ of $\mathrm{X}$, such that: $y \in u$ and $p \subset v$.

Definition $2.2 \mathrm{X}$ is lindeloff: If for each open covering $\mathrm{A}$ of $\mathrm{X}$, there exists an countable open subcovering of A.

Definition $2.3 \mathrm{Y}$ is regular in $\mathrm{X}$ : If for each $\mathrm{y}$ of $\mathrm{Y}$ and each closed subset $\mathrm{p}$ of $\mathrm{X}$, such that $y \notin p$, there are disjoint open subsets $\mathrm{u}$ and $\mathrm{v}$ of $\mathrm{X}$, such that: $y \in u$ and $p \cap Y \subset v$. 
Definition 2.4 $\mathrm{Y}$ is strongly regular in $\mathrm{X}$ : If for each $\mathrm{x}$ of $\mathrm{X}$ and each closed subset $\mathrm{p}$ of $\mathrm{X}$, such that $y \notin p$, there are disjoint open subsets $\mathrm{u}$ and $\mathrm{v}$ of $\mathrm{X}$, such that: $x \in u$ and $p \cap Y \subset v$.

Definition $2.5 \mathrm{Y}$ is countable1-paracompact in $\mathrm{X}$ : If for each countable open covering A of $\mathrm{X}$, there exists an open family covering $\mathfrak{R}$ of $\mathrm{X}$, such that: $\mathfrak{R}$ refines $\mathrm{A}, \cup \mathfrak{R}=X$ and $\mathfrak{R}$ is locally finite at each y of $\mathrm{Y}$.

Theory 2.6 If $\mathrm{X}$ is regular. Then, $\mathrm{Y}$ is regular in $\mathrm{X}$.

Proof. Let $\mathrm{y}$ is a arbitrary points of $\mathrm{Y}$ and an arbitrary closed subset $\mathrm{p}$ of $\mathrm{X}$, such that $y \notin p$. Since $\mathrm{X}$ is regular, so there exist two disjoint open subsets $u_{1}$ and $v_{1}$ in X, such that: $y_{1} \in u_{1}$ and $y_{2} \in v_{1}$. Obviously, $y_{1} \in u_{1}$ and $p \cap Y \subset v_{1}$. This is, $\mathrm{Y}$ is regular in $\mathrm{X}$.

Theory 2.7 If $\mathrm{X}$ is regular and $\mathrm{Y}$ is Lindloff. Then, $\mathrm{Y}$ is in countable1-paracompact $\mathrm{X}$.

Proof. Let $A=\left\{u_{s}: s \in S\right\}$ is an countable open covering of X, and y is an arbitrary point of $\mathrm{Y}$. Then there is an $u \in A$, such that: $v \in u$. Since $\mathrm{X}$ is regular, so there exists an open set $v_{y}$ of $\mathrm{X}$, such that: $y \in v_{y} \subset \bar{v}_{y} \subset u$. So $X \backslash v_{y}$ is closed in $\mathrm{X}$ and which does not contain $\mathrm{y}$. By the Theory 2.6, $\mathrm{Y}$ is regular in $\mathrm{X}$, so, there are two disjiont open sets $t_{y}$ and $w_{y}$, such that: $y \in t_{y}$ and $X \backslash v_{y} \cap Y \subset w_{y}$. It is obvious that $\bar{t}_{y} \cap\left(X \backslash v_{y}\right) \cap Y=\varnothing$, so we can get: $t_{y} \cap Y \subset v_{y}$. Then for each arbitrary points y of Y, there is an open set $t_{y}$ of X, such that: $y \in t_{y} \subset v_{y}$, $t_{y} \cap Y \subset v_{y} \cap Y$. This is, $\mathfrak{R}=\left\{t_{y}: y \in Y\right\}$ is an countable open covering of $\mathrm{Y}$. And since $\mathrm{Y}$ is Lindloff, so there exist an countable subcovering $\Re_{1}=\left\{t_{y_{j}}: j=1,2 \cdots\right\}$. We may also assume that $v_{y_{j}}^{\prime}=v_{y_{i}} \backslash \bigcup_{i=1}^{j-1} \bar{t}_{y_{i}}$ : for each j. It is obvious that: $\mathfrak{R}_{2}=\left\{v_{y_{j}}: j=1,2 \cdots\right\}$ is an open family of $\mathrm{X}$ and which refines $\mathrm{A}$ and for each $y \in Y$, since $Y \subset \bigcup_{j=1}^{\infty} t_{y_{j}}$. So there exist the most smallest integer $\mathrm{j}$, such that: $y \in \bar{t}_{y_{j}}$, so, $y \in v_{y_{j}}^{\prime}$, this is $Y \subset \bigcup_{j=1}^{\infty} v_{y_{j}}^{\prime}$ Also since $Y \subset \bigcup_{j=1}^{\infty} t_{y_{j}}$, then there $t_{y_{i}}$ which contains y. And since $v_{y_{j}}^{\prime}=v_{y_{j}} \backslash \bigcup_{i=1}^{j-1} \bar{t}_{y_{i}}$, so when $j>i t_{y_{j}} \cap v_{y_{j}}^{\prime}=\emptyset$, then, $\mathfrak{R}_{2}$ is locally finite at each y of $\mathrm{Y}$. This is, $\mathrm{Y}$ is in countable1-paracompact $\mathrm{X}$.

\section{The Property of Relative Compactness under the Perfect Mapping.}

Some properties of topological spaces under the perfect mapping were given in (R.Engelking, 1997). In this part, I studied the property of countable1-paracompact under the perfect mapping, and gave a result about it.

Definition $3.1 \mathrm{f}: X \rightarrow Y$ is a perfect mapping: If $\mathrm{f}$ is a continuous mapping which is closed and for each $y \in Y$, the fiber $f^{-1}(y)$ is compact subset of $X$.

Theorem 3.2 Let $\mathrm{f}: X \rightarrow Y$ is a perfect countable 1 mapping. If $Y_{1}$ is countable 11-paracompact in $\mathrm{Y}$. Then, $f^{-1}\left(Y_{1}\right)$ is countable 1-paracompact in $\mathrm{X}$.

Proof. Let $A=\left\{u_{s}: s \in S\right\}$ is an countable open covering of $\mathrm{X}$. Since $\mathrm{f}$ is a perfect mapping, so for each $y \in Y$, the fiber $f^{-1}(y)$ is a compact subset of X. Thus, there exists a finite subset of $s(y) \mathrm{S}$, such that: $f^{-1}(y) \subset$ $\bigcup_{s \in S(y)} u_{s}=u_{y(s)}$. Since $\mathrm{f}$ is a perfect mapping, by the TH1.4.13 in (R.Engelking, 1997), there exists an open neighborhood $w_{y(s)}$ of $\mathrm{y}$, such that: $f^{-1}(y) \subset f^{-1}\left(w_{y(s)}\right) \subset u_{y(s)}$. We may also assume that: $f^{-1}\left(w_{y(s)}\right)$ is $v_{y(s)}$. That is $v_{y(s)}=f^{-1}\left(w_{y(s)}\right)$. Then, it is obvious that: $v_{y(s)}$ is open in $\mathrm{X}$ and such that: $f^{-1}(y) \subset v_{y(s)}=$ $f^{-1}\left(f\left(v_{y(s)}\right)\right) \subset u_{y(s)}$ and is an open subset of Y. Obviously, $\mathfrak{R}_{1}=\left\{f\left(v_{y(s)}\right): y \in Y\right\}$ is an open covering of Y. Since $Y_{1}$ is nearly1-paracompact in $\mathrm{Y}$, so there exists an open family covering $\mathfrak{R}_{2}=\left\{v_{a}: a \in A\right\}$ of $\mathrm{Y}$ by open subsets of Y, such that: $\mathfrak{R}_{2}$ refines $\mathfrak{R}_{1}, \cup \mathfrak{R}_{2}=Y$ and $\mathfrak{R}_{2}$ is locally finite at each $y \in Y$. We may also assume that $f\left(v_{y(s)}\right)$ which contains $v_{a}$ is $f\left(y_{a}(s)\right)$. Since $\mathrm{f}$ is perfect mapping, thus, $\Re_{3}=\left\{f^{-1}\left(v_{a}\right), a \in A\right\}$ is an open family of $\mathrm{X}$ and locally Finite each $x \in f^{-1}\left(Y_{1}\right)$. Obviously. Let $\mathfrak{R}=\left\{f^{-1}\left(v_{a}\right) \cap u_{s} \cap Y: a \in A, s \in S_{a}(y)\right\}$. Then, $\mathfrak{R}$ is an open family covering of $\mathrm{Y}$ and such that $\mathfrak{R}$ refines $\mathrm{A}, \cup \mathfrak{R}=X$ and $\mathfrak{R}$ is locally finite at each $x \in f^{-1}\left(Y_{1}\right)$. That is $f^{-1}\left(Y_{s}\right)$ is countable1-paracompact in $\mathrm{X}$.

\section{References}

A.V.Arhangel'skii. (1996). Relative topological properties and relative topological spaces. Topology and Appl. 20, 1-13.

A.V.Arhangel'skii and T.Nogura. (1998). Relative sequentiality. Topology and Appl. 82,49-58.

A.V.Arhangrl'skii. (2002). From classic topological invariants to relative topological properties. Scientiae 
mathematicae japonicae, 55, No.1, 153-201.

A.V.Arhangel'skiiandI.Ju.Gordienko. (1994). Locally Finite Topological Spaces. Questions and Answers in General Topology. 12:1.

Baturov D.P. (1990). Normality in dense subspaces of products. Topology and Appl.36, 111-116.

Gartside P and Aneirin G. (2000). Relative Separation Properties. Topology Atlas.

R.Engelking. (1997). General topology.Warszawa. (Chapter 1 ). 